\title{
Article \\ Analysis of Acoustic Characteristics under Battery External Short Circuit Based on Acoustic Emission
}

\author{
Nan Zhou ${ }^{1,2,3, *}$, Xiulong Cui ${ }^{4}$, Changhao Han ${ }^{5}$ and Zhou Yang ${ }^{1}$ \\ 1 School of Mechanical Engineering and Automation, Northeastern University, Shenyang 110819, China; \\ yangzhou@mail.neu.edu.cn \\ 2 Key Laboratory of Vibration and Control of Aero-Propulsion System, Northeastern University, \\ Shenyang 110819, China \\ 3 Vehicle Measurement, Control and Safety Key Laboratory of Sichuan Province, Chengdu 610039, China \\ 4 School of Mechatronics Engineering, Harbin Institute of Technology, Harbin 150001, China; \\ cuixiulong8985@163.com \\ 5 School of Mechanical Engineering, Beijing Institute of Technology, Beijing 100081, China; \\ chwyt516080282@163.com \\ * Correspondence: zhounan@me.neu.edu.cn; Tel.: +86-(24)-8369-1095
}

check for updates

Citation: Zhou, N.; Cui, X.; Han, C.; Yang, Z. Analysis of Acoustic Characteristics under Battery

External Short Circuit Based on

Acoustic Emission. Energies 2022, 15, 1775. https://doi.org/10.3390/ en15051775

Academic Editor: Daniel-Ioan Stroe

Received: 24 January 2022

Accepted: 25 February 2022

Published: 28 February 2022

Publisher's Note: MDPI stays neutral with regard to jurisdictional claims in published maps and institutional affiliations.

Copyright: (C) 2022 by the authors. Licensee MDPI, Basel, Switzerland. This article is an open access article distributed under the terms and conditions of the Creative Commons Attribution (CC BY) license (https:// creativecommons.org/licenses/by/ $4.0 /)$.

\begin{abstract}
The safety of power batteries has received more and more attention in promoting electric vehicles. The external short circuit is particularly prominent as an abnormal and harmful event of a battery, and the exploration of in-situ low-cost detection technology for such an event is the starting point of this paper. By building an experimental bench that could detect the external short circuit of the battery and obtain the acoustic, electrode, and temperature responses, the resulting acoustic analysis would establish an internal connection with the electrode and temperature measurement when the external short circuit occurs. The respective acoustic response characteristics of different initial battery states of charge were analyzed by selecting appropriate acoustic characteristic parameters in the time and frequency domains. The acoustic measurement could represent the battery abnormality synchronously like the electrode measurement, and the results of the damage and rearrangement of the internal of the battery are easy to characterize through a moderate amplification of the acoustic response. The different initial state of charge (SOC) state reflects noticeable differences in the acoustic characteristics. Therefore, it is considered that the acoustic emission technology might have potential battery condition assessment capabilities and be a tool for in-situ battery fault diagnosis.
\end{abstract}

Keywords: lithium-ion battery; acoustic emission; state of charge; external short circuit; acoustic characteristic

\section{Introduction}

With the vigorous development of lithium battery chemistry technology, pure electric vehicles (PEVs) have gradually begun to replace fuel vehicles. Lithium batteries are widely used in electric vehicles due to a good balance of many aspects, such as cost, capacity, safety, and other excellent characteristics. People have anticipated higher requirements for the overall performance indicators of lithium batteries, especially in terms of volume/massenergy density, power density, life cycle, cost, safety performance, etc.

Battery-embedded sensors are used to collect valuable data to optimize battery operation strategies [1,2]. The data will help the battery management system to acquire the correct status of the battery, optimize the battery performance, and detect abnormal conditions of the battery in time. At the same time, from the perspective of vehicle battery driving range and power requirements, the demand for the number of lithium-ion batteries is increasing, and the performance of these batteries, such as fast charge and discharge capabilities and safety issues [3-5], are also constantly improving. To meet the needs of the market, enhance the performance and safety of batteries, and shorten the development 
cycle of new battery systems, it is necessary to carry out failure analysis and fault diagnosis of lithium batteries in the course of battery operation. The diagnostic techniques for Li-ion batteries are increasingly emphasizing a balance of economy and reliability due to their need to target larger battery sizes and the cost of batteries deployed in increasingly expensive applications, including electric vehicles.

\subsection{Literature Review}

Lithium-ion battery cells usually have variable electrical characteristics and are very sensitive to operating and environmental temperatures. With diagnostic tools and sophisticated battery management strategies, battery performance has been improved by confining battery operation within specified limits [6-8]. Those limits balance the battery's performance and alleviate the problem of rapid degradation. The causes of rapid degradation include high-current charging and discharging, low-efficiency temperature environments, and low or high battery charge timing. The state of the lithium battery would affect the life of the battery itself and be directly linked to the performance of the vehicle. The characteristic response of the battery electrode as a real-time diagnostic tool usually involves complex algorithm processing, and the high-precision hardware sensors for the battery management systems (BMS) are expensive. In this case, although the detection of electrode characteristics could identify abnormal data, it is challenging to locate the specific fault location, especially when the batteries are arranged in groups or packages. It can be used as a supplement to the judgment of battery failure by combining visualizing the inside of the battery. In recent years, acoustic-related testing methods have gradually gained more and more attention in lithium battery fault diagnosis due to their versatility, ease of operation, and acceptable accuracy of results.

In situ and ex situ diagnostic techniques have been broadly used in the fabrication, planning, performance assessment, and tracking of electrochemical devices $[9,10]$. There are four main methods for measuring lithium-ion battery (LIB) failure [11], including destructive testing, imaging methods, methods based on battery swelling, and methods based on acoustic measurements. The experimental study of lithium-ion battery failure is primarily destructive testing, which means that the battery is damaged or severely affected in its operational behavior and safety; it is crucial for the study of the mechanical and electrochemical relationships of the battery, but it rarely applies to troubleshooting.

Imaging methods such as X-ray or computed tomography are used to gain insight into the physical structure of a battery or material and can measure layer thickness through changes in the state of charge (SOC) or state of health (SOH) and identify structural defects in materials and battery components. Historically, X-ray tomography has been commonly used to obtain 3D images of batteries [12-14]. The introduction of lock-in thermal imaging technology also enables internal visible battery inspections. Combined with the thermal imaging technology developed in recent years, a clearer view of the internal failure of the battery would be achieved [15]. However, this usually requires a lot of laboratory equipment, space, and funds, so there is a high threshold for promotion and application. Another limitation is that the components to be tested must absorb X-rays to ensure the feasibility of detection $[16,17]$.

The research conducted by the swelling method is mainly based on the contact measurement for the pouch battery. It is an economical way to reveal most of the phenomena in LIBs and is widely used in laboratory-stage research. The research includes the correlation of SOC and charge-discharge $\mathrm{C}$ rate, considering the battery structure, battery $\mathrm{SOH}$ estimation, and the effect of temperature on swelling, etc. [18]. There is no report on the measurement of swelling under abuse conditions. Experimental modal analysis (EMA) is a relatively new topic in LIB measurements. No firm conclusions could yet be drawn about $\mathrm{SOC}, \mathrm{SOH}$, and other effects on EMA behavior, but can be used as an integrated diagnostic development tool.

A class of acoustic-based methods transmits mechanical waves into the battery cell through an actuator, and the receiver sensor measures the response of the device to an 
external stimulus, called ultrasonic testing (US) [19]. During scanning, the distance between the transducer and the object's surface to be measured remains constant. The energy of sound waves decays faster in gas than in liquids and solids, which will lead to low accuracy of measurement data. To enhance the transfer energy of sound waves in the sample and avoid external interference of the measured object, both the transducer and the battery are usually invaded into the coupling medium (gel or water) to reduce the effect of reflections at the outer interface on the measurement. The US can detect not only operating values, such as SOC and SOH, but also abusive behaviors, such as high temperature, electroplating, and overcharging, and applies to a wide range of frequencies and various form factor studies $[20,21]$. As a non-destructive battery diagnostic tool, some literature has explored acoustic methods to characterize Li-ion pouch cells, with sound as an indicator of battery structural health and state-of-charge-related parameters. Time of fly (TOF) offset and total signal amplitude as index parameters for ultrasonic analysis are used to predict the battery state and state of health. Combined with traditional electrode detection data, TOF could present direct structural information of actual batteries in real-time [22,23]. At the same time, many macroscopic-level static strains of batteries have been associated with the operating state in the battery, which shows a strong correlation with the volume change in some pouch batteries. Acoustic measurements have also been used to detect possible macroscopic crack detection, and microscopic measurements have been used to further clarify the cause of the shock response in the electrochemical reaction of the battery [24,25]. However, critical explanations for battery fault diagnosis and acoustic detection feature extraction are still lacking.

Acoustic Emission (AE) is another acoustic-based measurement method that differs from ultrasonic testing in that the object to be measured is not subjected to external mechanical excitation, but the material itself is measured by a connected (surface) sensor collecting fast releasing mechanical stress. The resulting sound waves are widely used to monitor fatigue, crack formation, and mechanical damage in building materials. Also included under external stimuli (e.g., changes in pressure, strain, temperature, or load), local sources within the material trigger energy release in the form of stress waves, which can be detected and converted on the surface of the measured object as electrical signals for subsequent studies [26,27]. Therefore, the use of AE to detect the electrochemical process of the battery is also a further application of its technical characteristics. For LIB batteries, the particle fracture will inevitably fluctuate in acoustic signals, which can be obtained theoretically through the selection of appropriate equipment and the tuning of filtering algorithms. However, it is accompanied by an increase in acquisition cost and higher requirements for robustness. Therefore, from the perspective of balancing the cost of signal acquisition and the ability to predict abnormal events, the recognition object of the acoustic signal may also need to be carefully selected. Potential sources of AE events in LIBs include electrode cracking, transition metal dissolution, cathode electrolyte interface (CEI) formation, solid electrolyte interface (SEI) formation, etc. On a cylindrical 18,650 cell, some researchers measured AE during full cell discharge [28], detected $\mathrm{SOH}$ by $\mathrm{AE}$ [29], and observed the trend of the AE intensity for each window as a function of a cycle. Other researchers used the AE to probe the generation, change process of solid electrolyte interphase (SEI) in batteries [30]. The acquired AE signals could also be divided into various types. Targeting for different analysis purposes, appropriate characteristic indicators should be selected carefully to explore the damage mechanism of lithium-ion batteries [30,31]. Generally speaking, with the occurrence of electrochemical reactions inside the battery, the transformation of almost any energy level will be associated with the rearrangement of the local structure. An acoustic signal will inevitably be emitted in this rearrangement process, which also means that $\mathrm{AE}$ can be regarded as a relatively simple principle diagnostic tool on the one hand; on the other hand, their broad applicability is accompanied by high sensitivity issues. At the same time, according to the different anode and cathode material selection and rational design processes mentioned in the literature [32], it is necessary to fully understand the acoustic characteristics of a single structure or component of the battery. In the future, in 
the face of acoustic events and characteristics with low discrimination, it is necessary to introduce more acoustic sensors for simultaneous observation of anodes and cathodes, and the introduction of half-cell will also be an ideal research object.

In general, a variety of battery fault diagnosis methods have been widely used in the research of LIB batteries, not only in the analysis of the regular operation of the battery but also in the real-time monitoring of various failure precursors. Destructive testing means that the battery is damaged or seriously affected in its operating behavior and safety. It is primarily used in laboratory research and unsuitable for in situ detection needs. It is of great help to study the mechanical and electrochemical relationship of batteries in the lab, while it is not applicable for troubleshooting in reality. Generally speaking, non-destructive testing (NDT) is of great significance for verifying battery quality and safety standards [33]. The detection of the swelling method has a good effect on a specific pouch battery. When the measured object is a prismatic battery or a cylindrical battery, the swelling application effect will be significantly reduced. The other three methods are classic non-destructive methods, which would provide potentially practical information and has the possibility of application in in-situ and ex-situ operations, helping to overcome the difficulties and the problems to be solved in traditional battery state estimation and fault diagnosis operations based on electrode measurements. Acoustic-based sensing technology is mainly used for SOC and SOH measurement of batteries, but the associativity of temperature, $\mathrm{C}$ rate, and abuse conditions are less considered. In terms of monitoring time, in comparison, imaging methods require less measurement time, but it is not easy to form a long-term continuous analysis process. Acoustic measurement has excellent advantages in long-term monitoring data accumulation. On that basis, deep learning and mathematical statistics have great potential as data-driven estimation methods, and they work best when combined with traditional electrode measurements such as voltage and current observations. So, the acoustic measurement is of great significance for battery state estimation and fault diagnosis based on battery electrode measurements.

\subsection{Motivation and Original Contribution}

From the perspective of battery safety, the short circuit caused by external circuit failure is a relatively severe electrical abuse behavior. The subsequent performance failure of the battery is easy to generate, which is reflected in capacity attenuation, short cycle life, poor consistency, easy self-discharge, high- and low-temperature performance degradation, etc.; on the other hand, the battery is prone to safety risks such as thermal runaway, gas leakage, and expansion deformation. From an acoustic point of view, the microstructure of the battery must be accompanied by relatively drastic changes in the short-circuit process of high current discharge, which is reflected in the transformation of the microstructure of the cathode and anode materials, breakdown of the separator accompanied by continuous heat release of the battery, temperature rise, and other behaviors. Changes in the micromechanical structure of the battery will negatively affect the current state and subsequent performance of the battery. Although the data measured by the electrode can detect the abnormality of the current data, the ability to capture some short-term abnormal signals is weak, and the subsequent impact cannot be continuously monitored and identified. For the short-circuit behavior of different initial SOC, the evaluation method should also require diversified measurements. In terms of the acquisition of acoustic signals and analysis of parametric variables, few studies have conducted an in-depth analysis of the high current caused by short circuits. Therefore, this paper analyzes the acoustic response characteristics of the battery cells with external short circuits (ESC) under different SOC initial conditions, obtains the acoustic discrimination criteria for the short-circuit fault diagnosis of 18,650 batteries, and conducts a possible correlation analysis. The research would provide a method for the failure analysis of lithium batteries, also a new promising tool. 


\subsection{Configuration of This Paper}

The remainder of this paper is organized as follows: Section 2 illustrates the experiment's setup. Preliminary Analysis of Acoustic Signals introduces the primary methods for this study. Results and discussion present the results of the analysis for the acoustic response of the external short-circuit battery and develop the necessary discussions. Finally, the conclusion of our approach is given in Conclusion. All term abbreviations used in the study could be found in Nomenclature.

\section{Experimental Section}

Acoustic measurements were performed on commercial 18,650-type cylindrical nickel manganese cobalt oxide (NCM) lithium cells. According to the test conditions and safety considerations, two cases of initial battery SOC of $80 \%$ and $50 \%$ were selected. When the battery is placed in the temperature chamber, the ambient temperature is set to $20^{\circ} \mathrm{C}$ to facilitate later observation of temperature changes and establish the connection between temperature, electrode measurement, and acoustic response. The battery-cell characteristics are shown in Table 1. Taking Lithium NCM111as an example, the electrochemical reaction during the charging and discharging process is shown in (1). Figure 1 shows the installed lithium-ion battery external short-circuit the experimental system. The computer is connected to the Motohawk via controller area network (CAN) and controls the relays in the circuit. Hall current sensors are used to measure short-circuit current. The ultrasonic needle sensor is from ndtXducer, and the miniature needle-type sensor is for industrial and laboratory use in ultrasonic and acoustic measurements with characteristics such as low cost, flat sensitivity, and good directivity, and its maximum operating temperature is around $100^{\circ} \mathrm{C}$. The sensor is used to collect the vibration signal generated by the change of the internal structure of the battery, and the output signal is a high-frequency voltage signal. The voltage, current, and vibration signals were all recorded by the Nioki MR6000 data acquisition device, and the short-circuit duration was $150 \mathrm{~s}$. To reduce the external interference to the acoustic signal of the battery, a vibration isolation material is arranged around the battery and the acoustic sensor; to enhance the sensor's ability to receive signals, the Vaseline gel is applied at the contact position between the battery body and the sensor.

$\mathrm{LiNi}_{\frac{1}{3}} \mathrm{Co}_{\frac{1}{3}} M n_{\frac{1}{3}} \mathrm{O}_{2} \underset{\text { discharge }}{\stackrel{\text { charge }}{\rightleftarrows}} \mathrm{Li}_{1-x}\left(\mathrm{Ni}_{\frac{1}{3}-x}^{2+} N i_{x}^{3+}\right) \mathrm{Co}_{\frac{1}{3}} \mathrm{Mn}_{\frac{1}{3}} \mathrm{O}_{2}+x L i^{+}+x e^{-}\left(0 \leq x \leq \frac{1}{3}, 3.8-4.1\right)$

Table 1. List of main parameters of the experiment.

\begin{tabular}{cc}
\hline Type of the Battery Cell & 18,650-Type Cylindrical NCM Lithium Cells \\
\hline Nominal cell capacity $(0.3 \mathrm{C})$ & $2.0 \mathrm{Ah}$ \\
Average battery cell voltage & $3.6 \mathrm{~V}$ \\
End of discharge voltage & $2.5 \mathrm{~V}$ \\
High voltage protection & $4.2 \mathrm{~V}$ \\
Operation temperature range & $-20-55^{\circ} \mathrm{C}$ \\
Cathode materials & $\mathrm{LiNi}_{1-\mathrm{y}-\mathrm{z} \mathrm{Co}_{\mathrm{y}} \mathrm{Mn}_{\mathrm{z}} \mathrm{O}_{2}}$ \\
Anode materials & Graphite \\
\hline
\end{tabular}

The collected raw voltage, current, acoustic, and temperature signals are shown in Figure 2. The time range of the short-circuit occurrence can be clearly defined by the fluctuating region of the voltage change. The voltage has the remarkable characteristics of initial sag, fluctuation retention, and secondary sag. The corresponding current has a similar change process of the initial jump, mid-wave, and terminal drop, which is a phenomenon that can be clearly distinguished from the short-circuit condition at the electrode measurement. Corresponding to the temperature signal, the initial occurrence of the short circuit could obtain the rise of the temperature for the positive and negative electrodes and the slight decrease of the temperature after the short circuit is completed. Corresponding to the acoustic signal, the initial signal observation would roughly distinguish between 
the beginning and the end of the short circuit (compared to the range determined by the voltage or current); the signal has an initial apparent change (at the beginning of the ESC), and a relatively weak mutation (at the end of the ESC); other acoustic features are not very obvious; it is difficult to distinguish from the data on the figure.

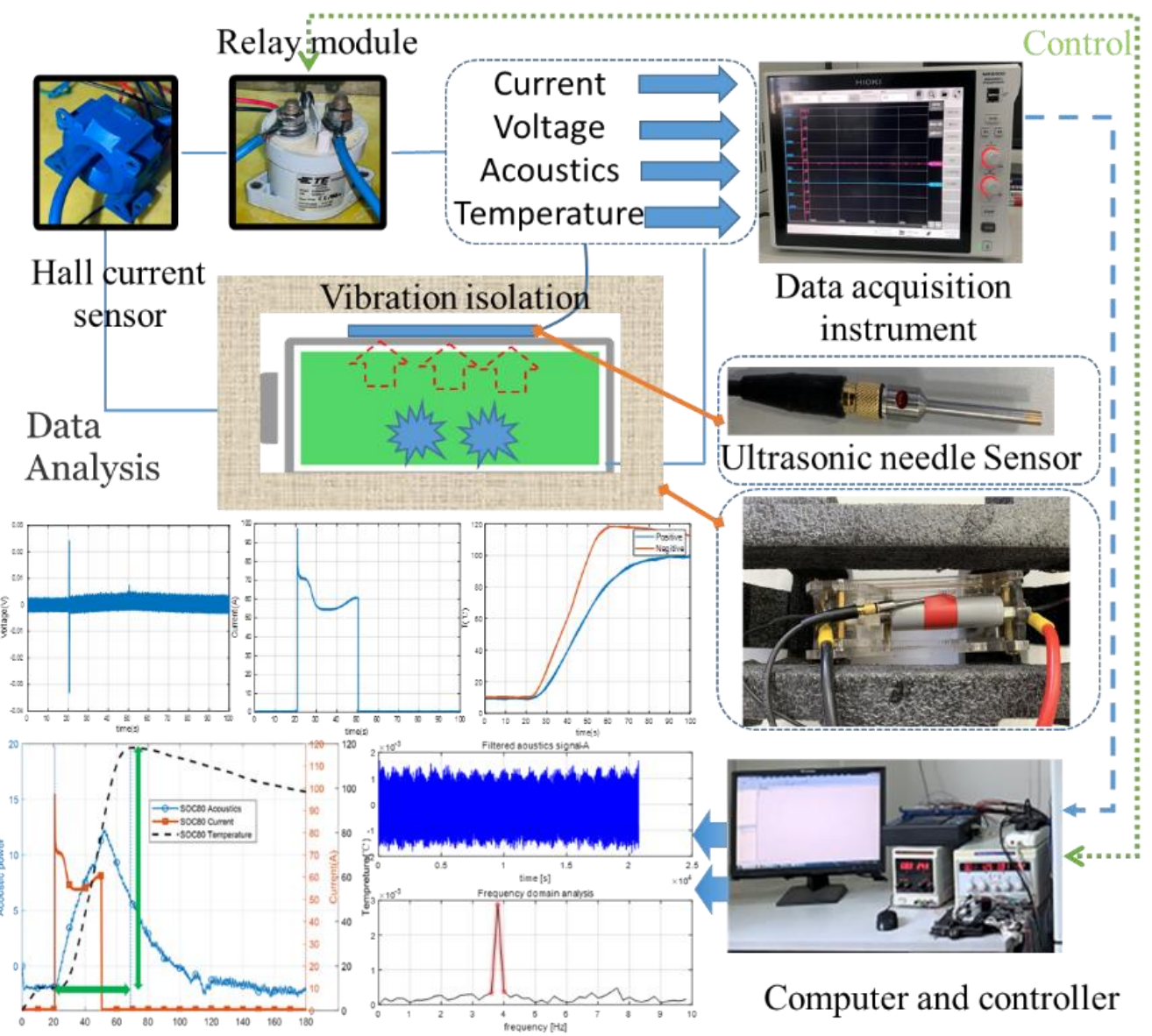

Figure 1. Schematic diagram of the ESC experimental setup based on AE.
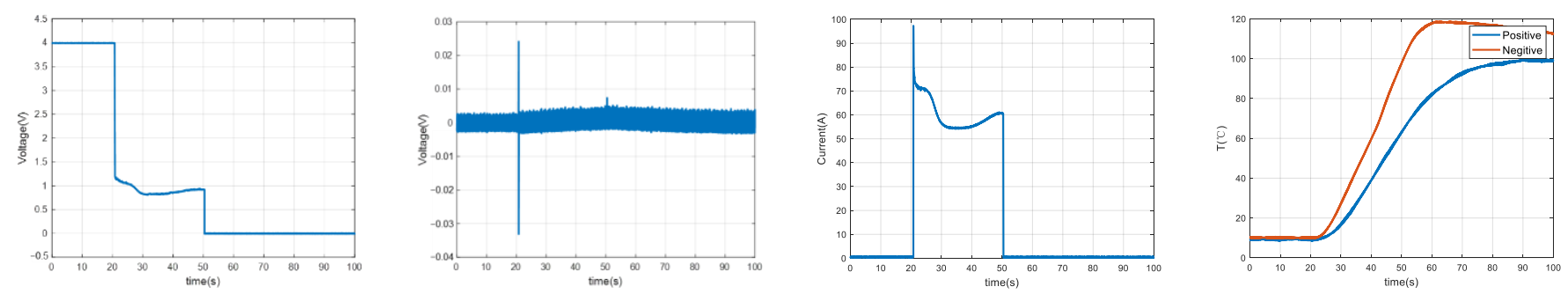

Figure 2. Voltage, current, acoustic, and temperature data curve across the entire ESC.

Therefore, in terms of acoustic signal data analysis, phase spectrum analysis reduces various influencing factors to obtain signal amplitude and phase values for further research. The frequency-domain characteristics of the AE signal were analyzed from the time-domain acoustic signal by Fast Fourier Transform (FFT). 


\section{Preliminary Analysis of Acoustic Signals}

According to the change of the corresponding current, the acoustic signal is preliminarily divided into four time slots in Figure 3, where A is before the ESC, B is the continuous process of the ESC, C is the near-end after the ESC, and D is the far end after the ESC, which are used to observe the possible acoustic responses of the battery after a short circuit occurs. The following results are obtained after filtering the PPT analysis for the acoustic characteristic data segment of the above time slot.

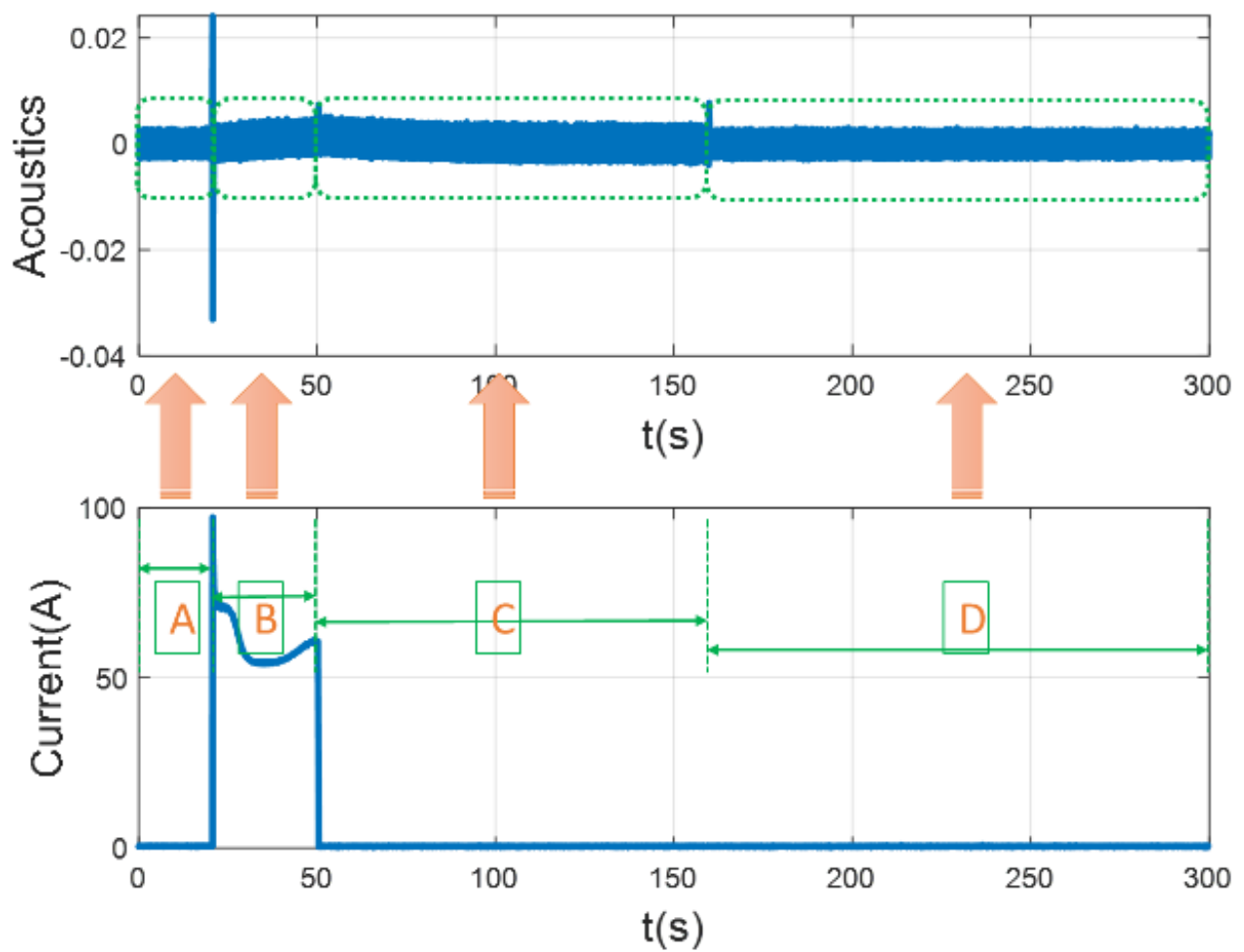

Figure 3. Segmentation diagram of an acoustic signal.

In Figure 4, there is an apparent difference between the amplitude change of shortcircuit occurrence and the short-circuit occurrence near-end, which reflects a difference value of more than ten times. From the perspective of frequency, the frequency of the inherent acoustic characteristics of the battery cell is relatively concentrated before the short circuit occurs, which is between $3-4 \mathrm{~Hz}$. The frequency characteristics of the ESC at the near-end are shifted to the left, indicating that the internal structure of the battery is changed due to the breakdown of the short-circuit current and the acoustic features are changed, and the material fracture continues after the ESC in the proximal short-circuit area C. It may be due to residual stress, which is reflected in the continuous increase in the amplitude of the center frequency. The continuous temperature rise also proves that the structure continues to change after the short circuit, and there is continuous heat release. The characteristics of the acoustic response of region $\mathrm{D}$ become blurred due to the rearrangement of the structure and lose its original characteristics, which is reflected in the chaos of the microstructure and change. These indicate a dramatic change in the mechanical properties of the electrode, which is consistent with the static mechanical analysis of the electrode $[24,26,30]$. 

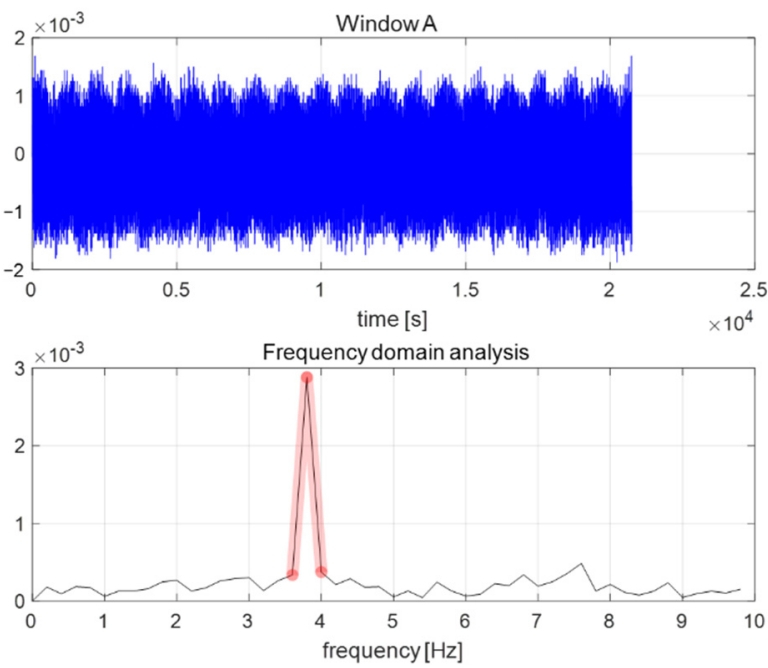

(A)
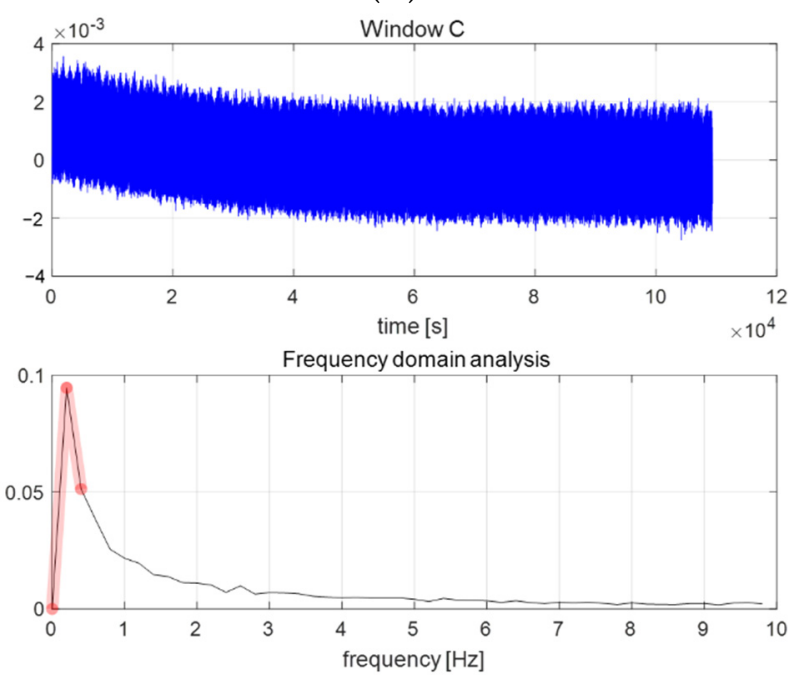

(C)
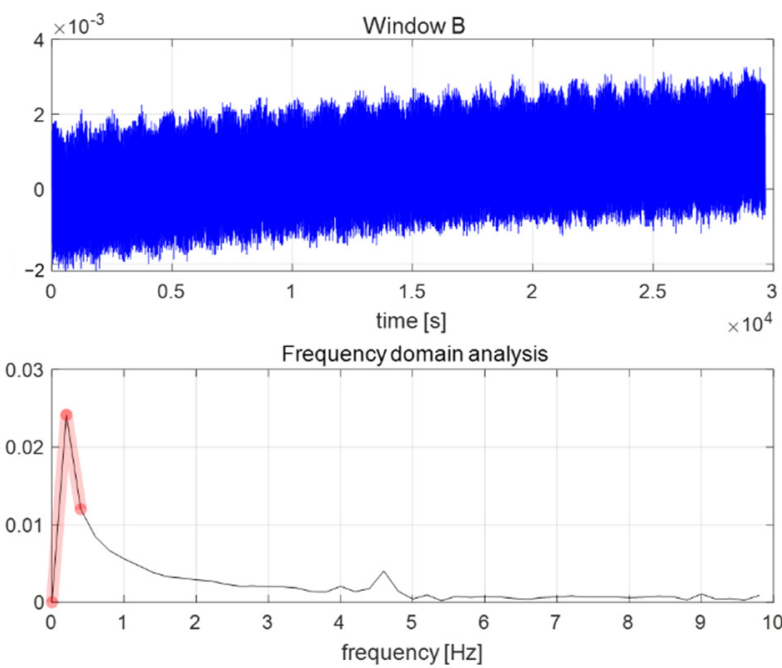

(B)
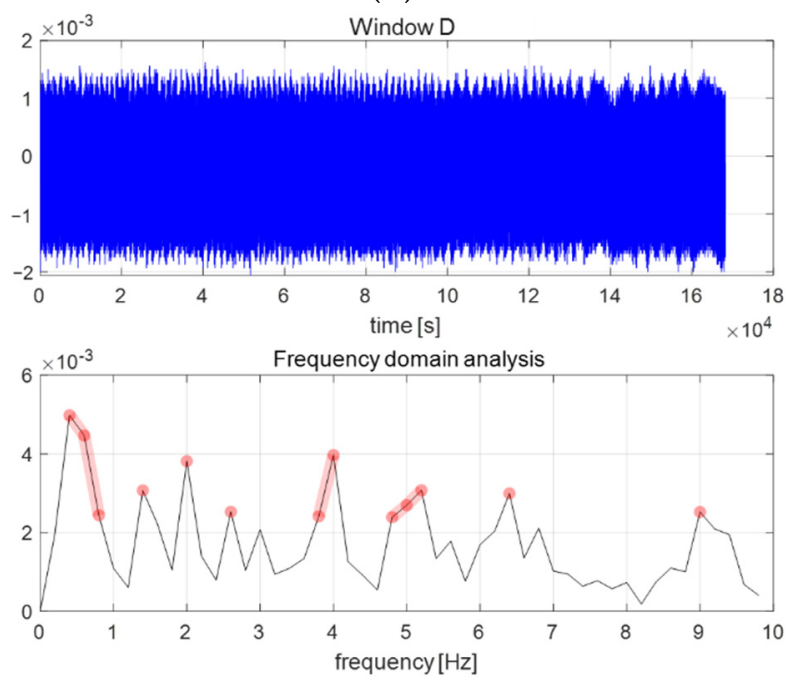

(D)

Figure 4. Original acoustic signal and frequency domain characteristics of window A-D. (A), Filtered acoustic signal before the ESC; (B), Filtered acoustic signal during the ESC; $($ C), Filtered acoustic signal near-end after the ESC; (D), Filtered acoustic signal far-end after the ESC.

From the perspective of the acoustic characteristics of a single short-circuit battery, in terms of the near-end frequency domain characteristics before, during, and after the ESC (window A-C), it is preliminarily shown that the value of the center frequency and its amplitude, which are representative, would characterize the change of the internal structure and the change of the mechanical properties when the short circuit occurs to a certain extent. The signal at the far (window $\mathrm{D}$ ) end becomes more ambiguous due to the internal change of its acoustic characteristics after the short circuit occurs so that it can be temporarily ignored in selecting the ESC data.

Therefore, in this paper, the correlation analysis of the measured values of electrodes, acoustics, and temperature is carried out in both time and frequency domains. According to the relationship between voltage, current, and temperature, the implication between the response of the battery in different time slots during the ESC process and the characteristic acoustic parameters would be obtained; the representation to the initial SOC at the occurrence of the ESC might be clear; while the link between cell structural damage and rearrangement and the acoustic response could be discussed. The overall framework of the presented ideas is shown in Figure 5. 


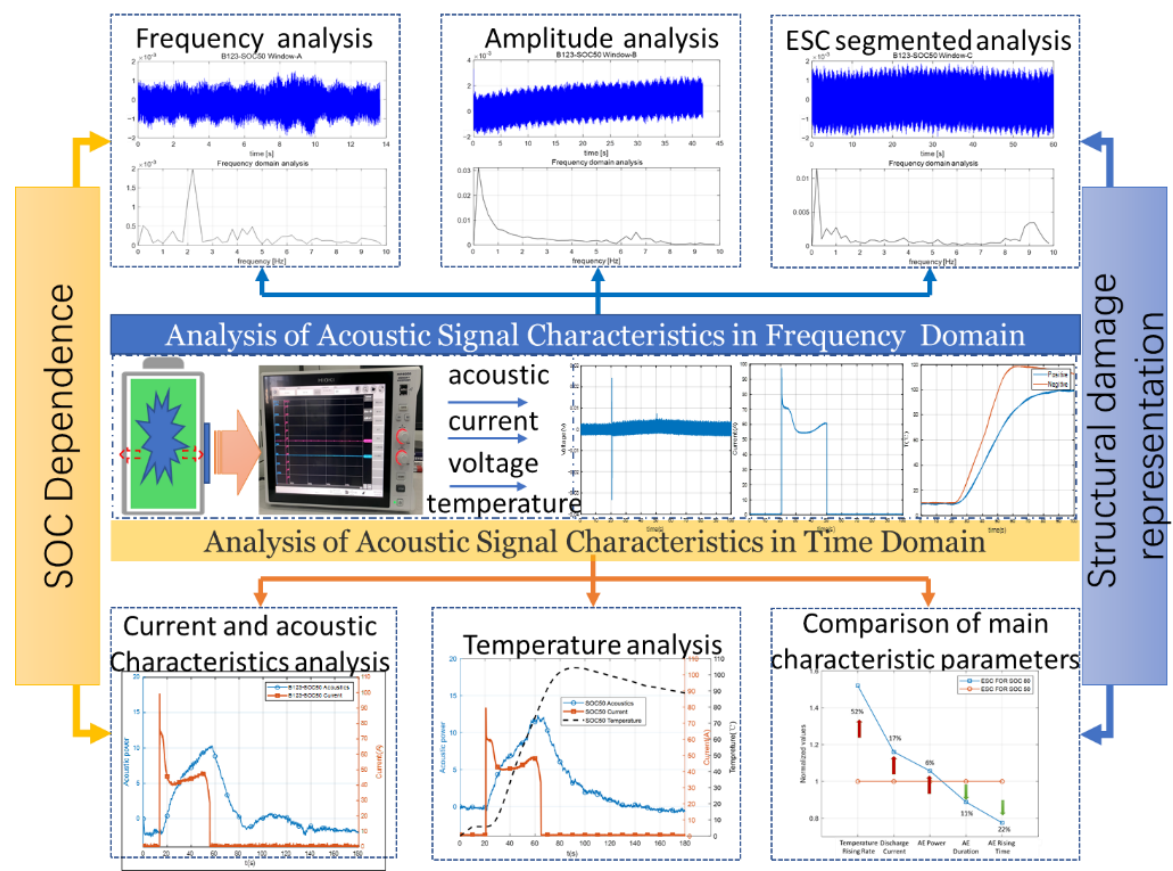

Figure 5. Framework for the acoustic analysis in ESC.

\section{Results and Discussion for Acoustic Characteristics of Battery Short Circuit under Different SOC Conditions}

\subsection{Analysis of Acoustic Signal Characteristics in Time Domain}

Characteristic parameters that are important for battery acoustics include rise time, the time between the first threshold crossing and the peak amplitude; "duration" or the time interval between the first and last threshold crossing in a burst signal; and the amplitude of the response acoustic intensity, etc. The preferred peak value (amplitude) reflects the response of the whole battery cell to the high-discharge current process in the short-circuit process, which is closely related to the damage to the battery's internal structure when the short circuit occurs. What the acoustic sensor would receive is damage to the internal structure of the battery. Although the specific position of the AE (sound radiation intensity) cannot be determined under passive conditions, its amplitude reflects the characteristics of the special battery discharge conditions. It would be used for comprehensive judgment of the battery state.

The original acoustic signal obtained by the sensor has a low degree of recognition with poor signal legibility. To better analyze the acoustic signal, the coaxial variation curve of the current and the acoustic signal is obtained after processing through a rational transfer function. The acoustic signal strength is normalized after filtering to ensure good readability of the values. Overall, in the time range of interest, regardless of the initial SOC (SOC80 OR SOC50) value, the acoustic signal immediately responds when a short circuit current occurs. Still, the trend of the acoustic signal is a step-by-step process, and its peak value shows at the end of the short-circuit process. In Figure 6, it appears later; that is, it could be found from the figure that the acoustic curve reaches its peak value in about $5 \mathrm{~s}$ after the ESC current drops to 0 . The current change is generally in a relatively short time, but the acoustic change starts at the same time as the current; while it is slightly achieved, a delayed peak point reflects that the rearrangement of the internal structure of the battery is the consequence of the short-circuit current shock. The subsequent decline process demonstrates the process of the destruction and rearrangement of the battery content structure. More importantly, from the perspective of detection, the initially short-term electrode behavior would be transformed into a more obvious acoustic change process, reflecting the vital characterization significance of the battery acoustic characteristics for the abnormal state of the battery. 

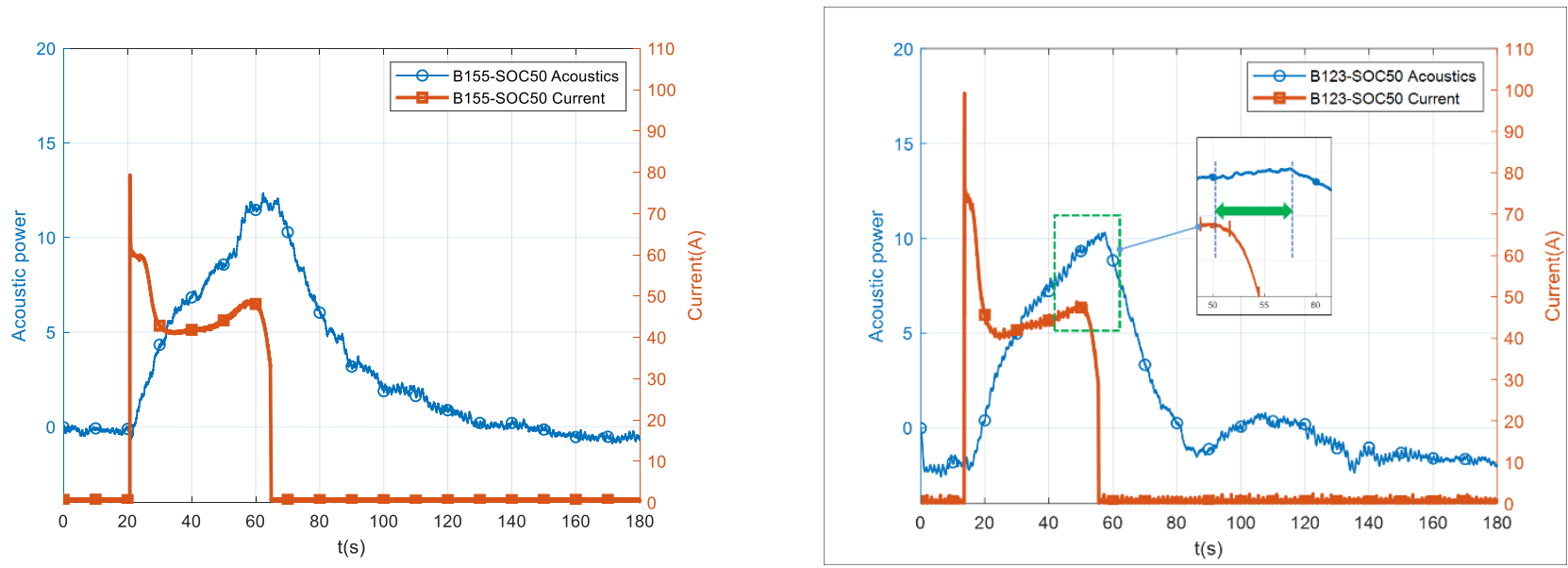

Figure 6. Current and acoustic characteristics of ESC under initial SOC50 condition.

Through observation for Figure 7, there is a specific SOC correlation between the acoustic intensities detected in SOC80 and SOC50. The acoustic radiation intensity of SOC 80 is higher than that of SOC50 by more than $6 \%$, which corresponds to the case of high current discharge. Compared with SOC50, the external short-circuit discharge current of SOC 80 also increases by $17 \%$. Of course, this is in line with our expectations for the initial state of the battery. For the analysis of battery AE duration, the structural characteristics of the battery itself reflect the response to the short-circuit process. Due to the difference in the battery's state, the degree of damage to the battery resulting from high-current discharge is also different. In Figure 8, SOC80 and SOC50, from the perspective of the two initial states, the acoustic response process of SOC 80 is shorter, reflecting the severity of the structural damage process. The $22 \%$ reduction for the AE rise time in SOC 80 also demonstrates the severity of structural damage inside of the battery, which does not optimistically speculate the possibility of structural rearrangement and repair. In the acoustic response of B123 battery SOC50 Figure 6, the fluctuation of the acoustic signal could be observed in the later stage, which may indicate the possibility of repair after structural damage. In the case of SOC80, no noticeable acoustic signal fluctuations were found subsequently.
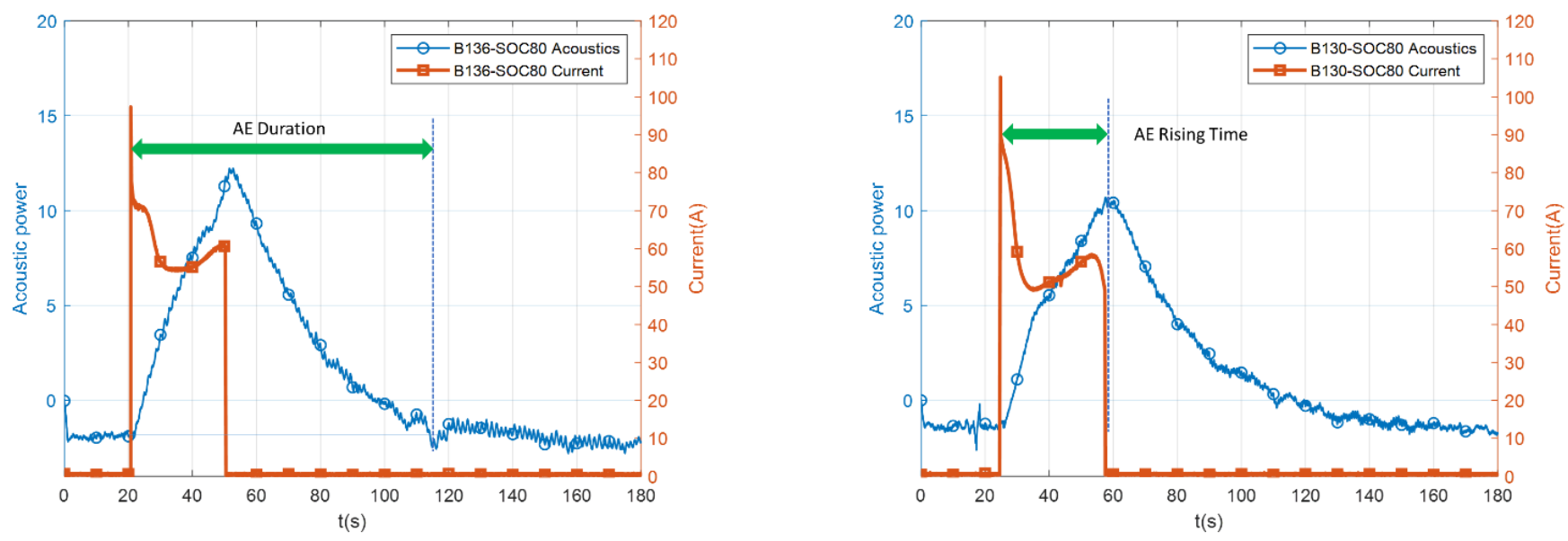

Figure 7. Current and acoustic characteristics of ESC with initial SOC80 condition. 

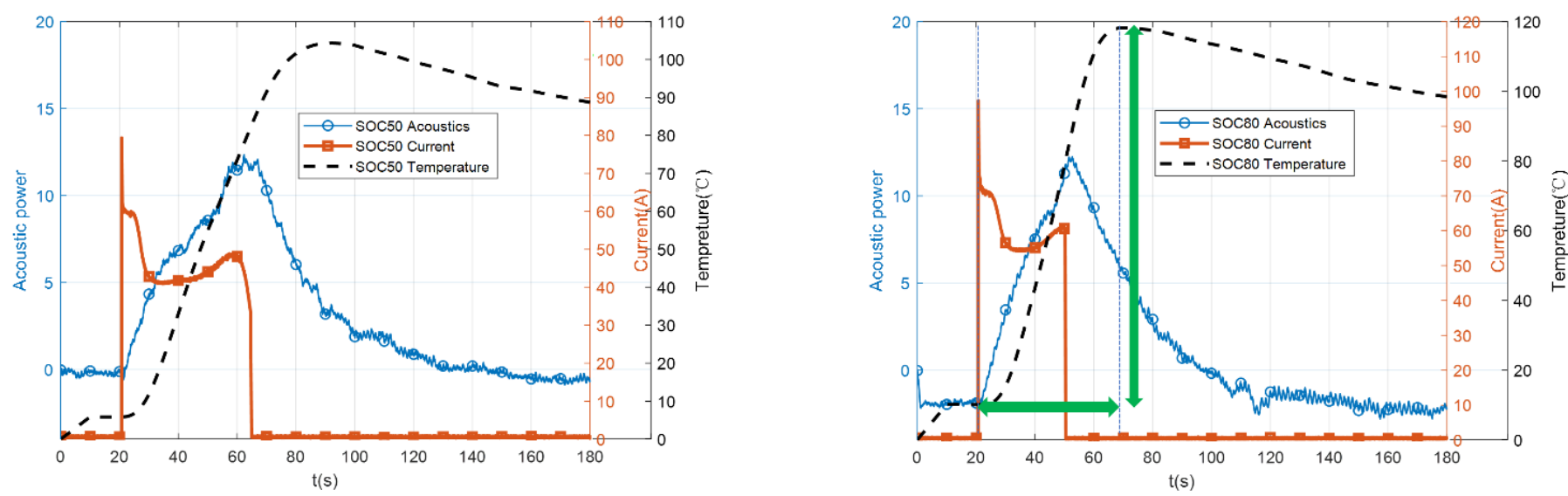

Figure 8. Current, acoustic, and temperature average characteristics of ESC under initial SOC50 and SOC80 conditions.

To observe the relationship between electrode measurement (current), temperature, and acoustic signal more intuitively, the data of SOC50 and SOC 80 are averaged to obtain a comparative analysis of coaxial curves under different SOC conditions, as shown in Figure 8. Finally, from the perspective of the temperature change during the short-circuit process, multi-position high-strength structural damage probably occurs inside the battery parallelly. So, the temperature rise rate for the case of SOC 80 is $52 \%$ higher than that of SOC50, while the time of its peak arrival is later than the acoustic peak. The reason for the subsequent temperature descent is more complicated. It is presumed to be the result of the combined effect of two factors: on the one hand, with the end of the primary short-circuit reaction, the large-scale structural damage stops; on the other hand, with some small-scale structural repairs, while some continuous destruction of the structural rearrangement results in the constant local release of heat, the observed result is a slower decline rate after the peak. Therefore, the judgment of the abnormal state of the battery from the perspective of temperature seems to have a relatively evident hysteresis in terms of timeliness, as the major damage to the internal structure of the battery has been completed. In this case, the analysis of the acoustic properties would present the abnormal behavior of the battery ahead of the temperature. Considering that the battery temperature will also be undulate due to heat accumulation during the regular operation of the battery, the direct response of the acoustic characteristics to the changes in the battery structure has certain advantages. The acoustic state can be used as the basis for judging the abnormal state of the battery. In Figure 9, all the values are normalized, and the SOC50 is used as the comparison benchmark, reflecting the comparison results of the above-selected parameters.

\subsection{Analysis of Acoustic Signal Characteristics in Frequency Domain}

The correlation analysis of the external short-circuit acoustic characteristics of the battery with two initial conditions of battery SOC of $80 \%$ and SOC of $50 \%$ was carried out, and three time windows (before, during, and after the ESC) were selected as the main observation time slot. Here, the magnitude of the amplitude in the frequency domain is used to represent the strength of the acoustic characteristics. 


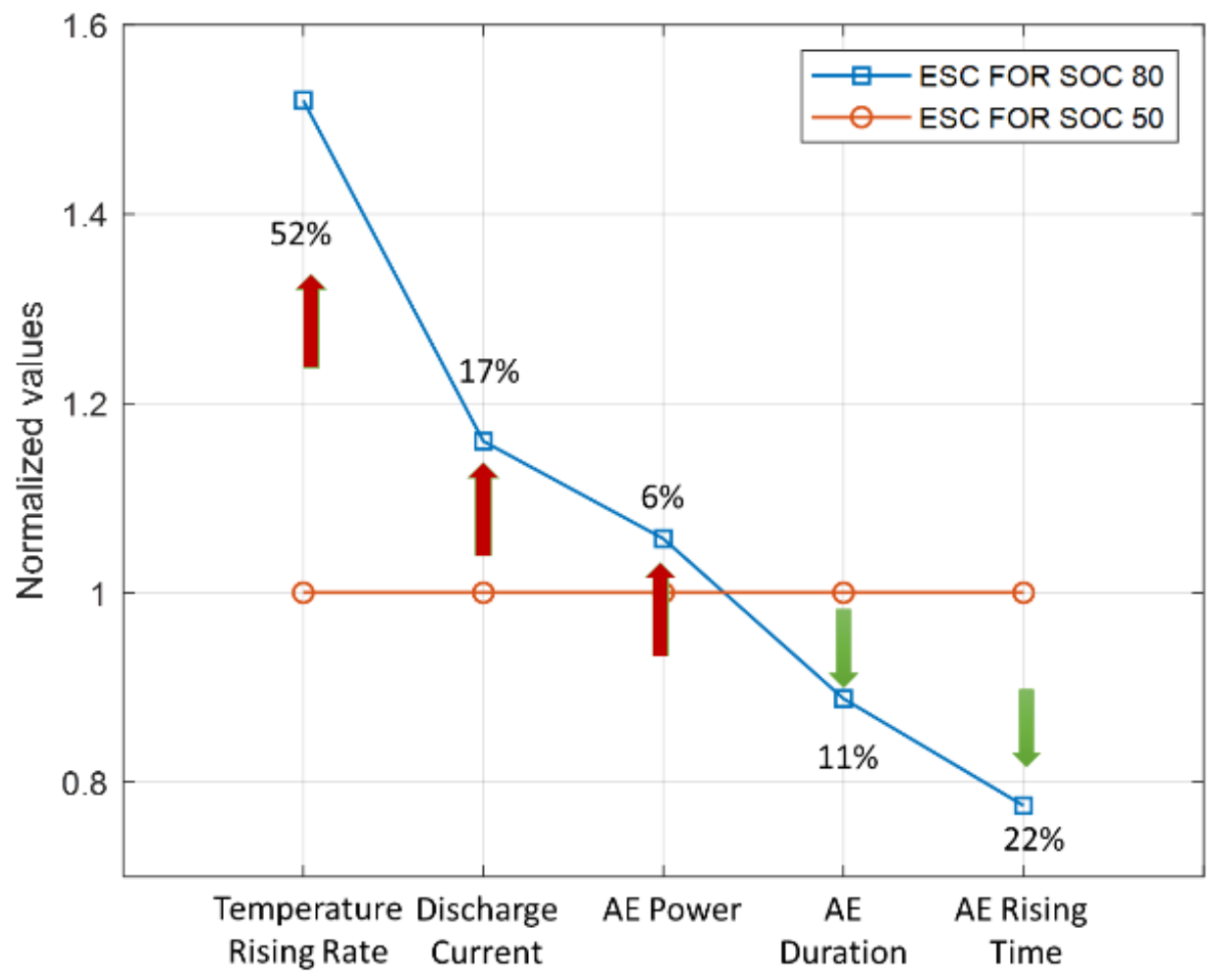

Figure 9. Comparison of main characteristic parameters of ESC occurrence.

In Figure 10, based on the acoustic signal characteristics in the three time windows concerned, the short-circuit acoustic features window-A of SOC80 Figure 10(a1, b1) generally shows relatively stable acoustic characteristics. The center frequency is between $3-6 \mathrm{~Hz}$, which refers to the background acoustic aspects of the operation of the experimental device. Depending on the arrangement of the device, it would be used as an initial calibration reference in the future considering improving the accuracy of the identification of the acoustic characteristics for different types of batteries or setups. In the acoustic characteristics of the window-B range Figure 10(a2, b2), the center frequency moves to the low-frequency area, and the amplitude is ten times higher than that of the window-A field. The recognition is with the remarkable feature, representing that the system structure responds strongly and concentratedly to ESCs, which may be due to the transformation of the internal structure when the high current, inducing the breakdown of the separator, or the structural rearrangement. The system is damaged, but the subsequent structure might continue to be damaged or maintained. It is necessary to move the data analysis of area $\mathrm{C}$. The acoustic characteristics of the window $\mathrm{C}$ interval do not change significantly from the perspective of the center frequency, but there are specific differences in the amplitude changes. A set of data shows that the $C$ interval in B130 Figure 10(b3) amplitude is unchanged, but the $C$ interval in B136 Figure 10(a3) data shows an increase of about five times the acoustic signal intensity, which indicates that even if there is no subsequent operation of the electrode terminal after the short circuit occurs, the trend of structural rearrangement and change continues after the ESC. The fluctuation may be different for individuals, but the trend should be the change of battery structure. As the current device cannot guarantee the safety of subsequent battery electrode tests after the ESC, further observation and analysis of the acoustic characteristics of the battery electrode operation are still to be confirmed, and more experimental data are needed. 

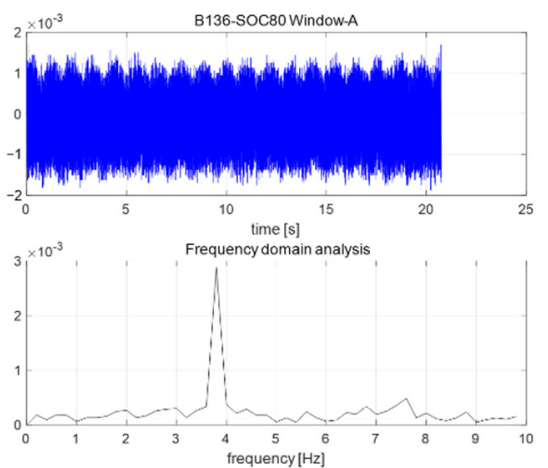

(a1)
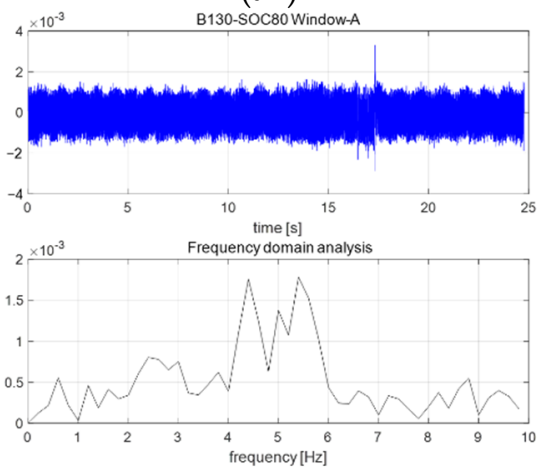

(b1)

(A)
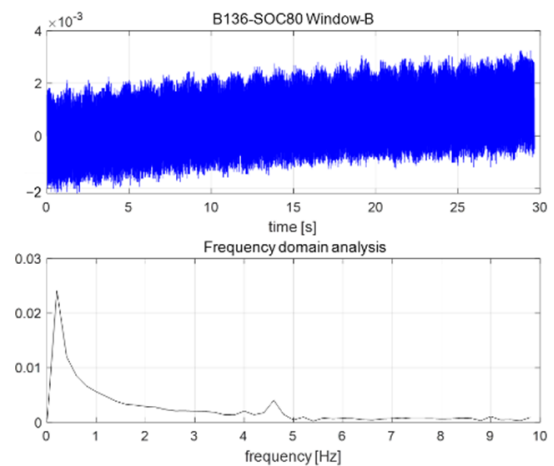

(a2)
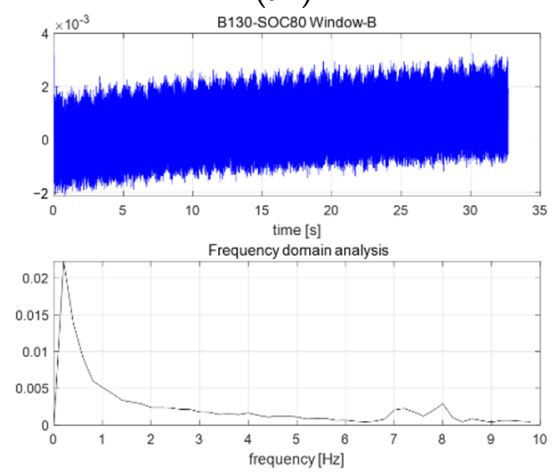

(b2)

(B)
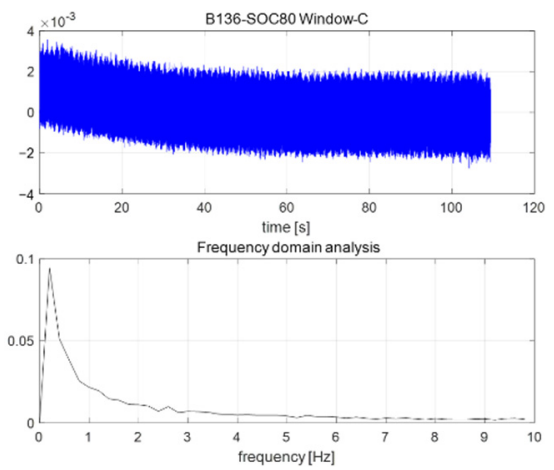

(a3)
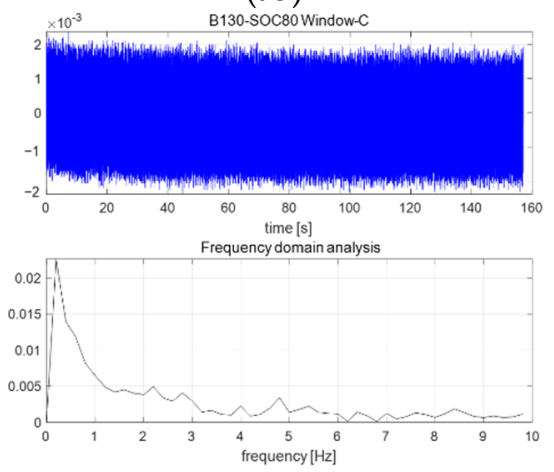

(b3)

(C)

Figure 10. Two cases frequency domain characteristics of window A-C with initial SOC80 condition. (A), acoustic signal before the ESC in (a1,b1); (B), acoustic signal during the ESC in $(\mathbf{a} 2, \mathbf{b} 2)$; (C), acoustic signal near-end after the ESC in $(\mathbf{a} 3, \mathbf{b} 3)$.

From the analysis of the initial battery short-circuit test results of SOC50, the overall trend is similar to that of SOC80. Still, the specific characteristics also demonstrate strong SOC dependence, in Figure 11. For example, the value of the center frequency moves along the low-frequency direction, the approximate frequency range is between $2-4 \mathrm{~Hz}$ Figure 11(a1, b1), and the amplitude of the center frequency is less than the case of SOC80, which reflects a relatively obvious SOC correlation. In the process of the ESC, that is, in window B Figure 11(a2, b2), the amplitude of its center frequency also appears to move to the low-frequency end, and the amplitude is lower than that of SOC80. Still, it has an increase of about 15 times compared with the amplitude of SOC50 window A. The shortcircuited near-end acoustic signal in window $C$ shows that the structure remains unchanged Figure 11(b3) or further aggravates the damage Figure 11(a3). Based on the observation of acoustic data, there is no observation that the acoustic characteristics represented by the structure would be restored to the initial state (window A) after a short circuit occurs. Even if it is left standing for a long enough time, no similar supporting data are observed. It is explained that the microstructure damage of the battery cells after the high current short circuit is irreversible, and it should be isolated or replaced in time from the perspective of battery safety. 


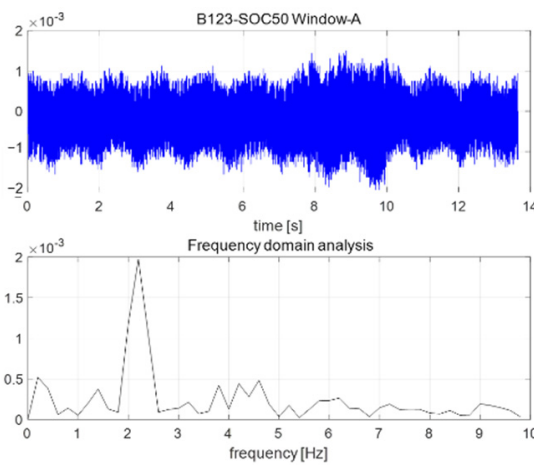

(a1)
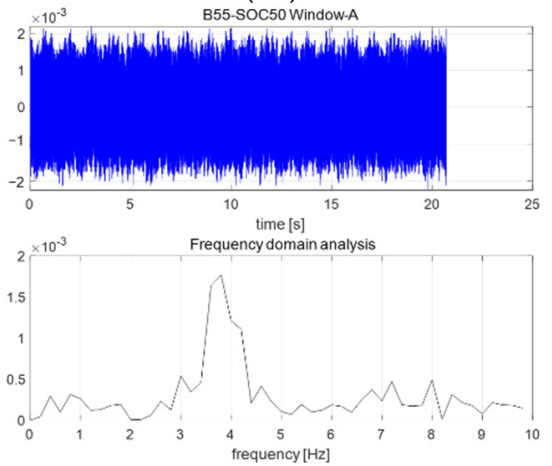

(b1)
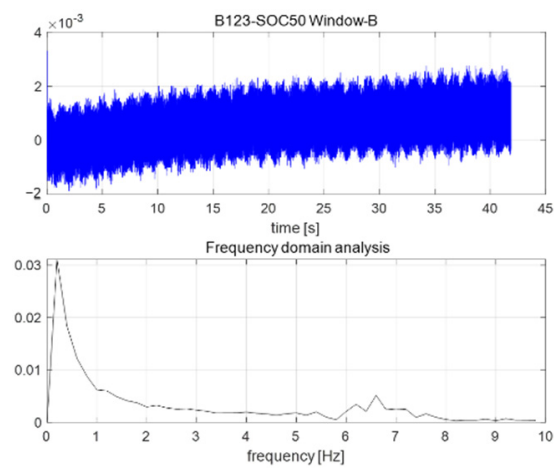

(a2)

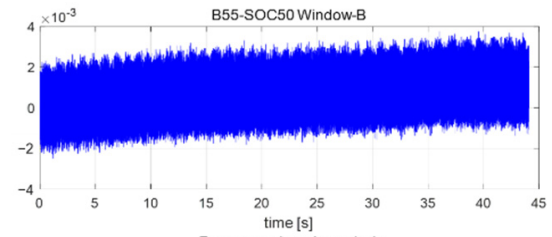

Frequency domain analysis

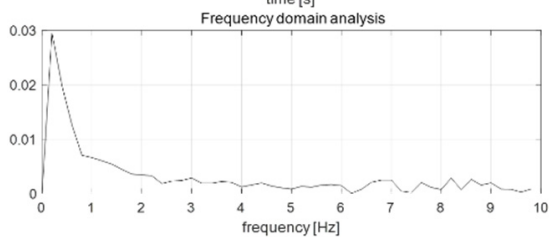

(b2)
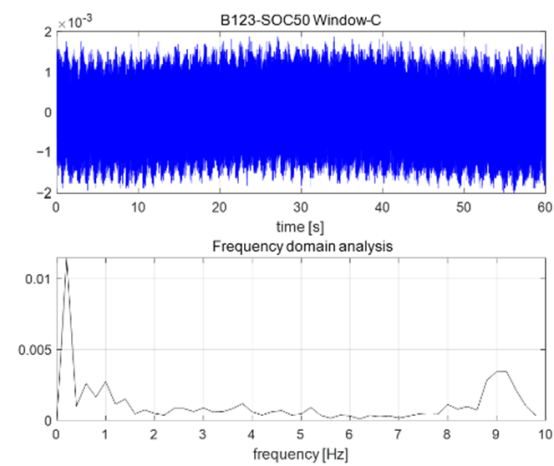

(a3)
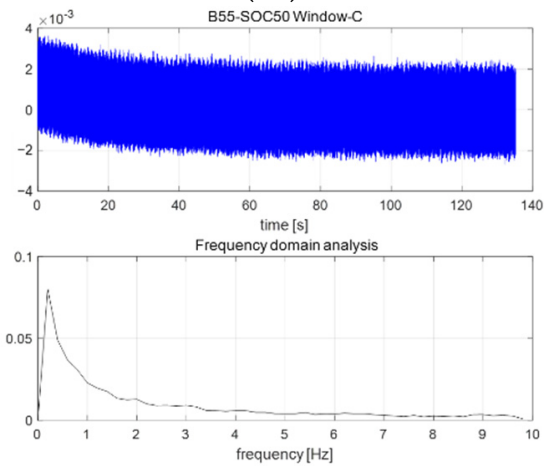

(b3)

(A)

(B)

(C)

Figure 11. Two cases frequency domain characteristics of windows A-C with initial SOC50 condition. (A), acoustic signal before the ESC in (a1,b1); (B), acoustic signal during the ESC in $(\mathbf{a} 2, \mathbf{b} 2)$; (C), acoustic signal near-end after the ESC in $(\mathbf{a} 3, \mathbf{b} 3)$.

\section{Conclusions}

This study investigates the AE characteristics of cylindrical lithium batteries when an external short circuit occurs. Firstly, a test bench capable of measuring the acoustic characteristics of the external short-circuit of the battery is built to obtain the electrode, temperature, and acoustic data of the battery when the ESC occurs. Then, the analysis is carried out from two aspects of the time domain and frequency domain. In the time domain, the acoustic signal of the battery appears synchronously with the voltage/current change during the ESC. With the destruction and rearrangement of the internal structure during the short circuit, the acoustic response gradually increases and reaches a peak after the end of the ESC. The critical acoustic characteristic values of rising time, amplitude, and duration were selected, the SOC correlation analysis was carried out for batteries with different initial SOC, and the mapping relationship with battery electrode and temperature characteristics was further considered. In terms of frequency domain analysis, the acoustic response of the battery is divided into three observation windows before, during, and after the ESC, and the center frequency and amplitude of the acoustic signal in the three stages are discussed. The correlation between the initial SOC of the short-circuit occurrence and the acoustic response is verified. The expectation of the SOC dependence in the time domain is confirmed. Therefore, by selecting appropriate acoustic characteristic parameters, $\mathrm{AE}$ technology has the ability to diagnose abnormal battery status together with the electrode and temperature measurement.

From the observation results of the ESC in analysis, the discharge current hopping from 0 to 20-30 C-rate, the change of the acoustic intensity could be observed, and there is still a lack of acoustic-related active constant discharge current test data at different levels to make further judgments. According to the results observed so far, the correlation between C-rate and acoustic energy is not clear, or the acoustic energy is relatively independent of the ESC in our research. Compared with electrode measurement, it would extend the duration 
of abnormal events and improve the detection rate of suspicious events. Compared with temperature measurement, AE can reflect the damage of abnormal circumstances to the structure earlier, to gain time for subsequent operations. In the future, combined with the analysis of the charge and discharge acoustic characteristics of different currents of the different SOC states, and supplemented by the correction of temperature effects, a more comprehensive battery acoustic and electrode characteristics would be established. The mapping relationship of characteristics will provide more basis for battery state estimation, life span estimation, and battery energy management.

Author Contributions: Conceptualization, N.Z.; methodology, N.Z.; software, N.Z.; validation, Z.Y., X.C. and C.H.; formal analysis, N.Z.; investigation, X.C. and C.H.; resources, X.C. and C.H.; data curation, X.C.; writing—original draft preparation, N.Z.; writing—review and editing, X.C. and C.H.; visualization, Z.Y.; supervision, N.Z.; project administration, N.Z.; funding acquisition, N.Z. and Z.Y. All authors have read and agreed to the published version of the manuscript.

Funding: This research was funded by the National Natural Science Foundation of China (52172401), Vehicle Measurement, Control and Safety Key Laboratory of Sichuan Province (QCCK2021-005).

Institutional Review Board Statement: Not applicable.

Informed Consent Statement: Not applicable.

Data Availability Statement: Please contact the corresponding author for the data from this study.

Acknowledgments: Acknowledge affiliation 2 to provide the site and some equipment for experiments.

Conflicts of Interest: The authors declare no conflict of interest.

\begin{tabular}{ll}
\multicolumn{2}{l}{ Nomenclature } \\
PEVs & Pure electric vehicles \\
SOC & State of Charge \\
BMS & Battery management system \\
LIB & Lithium-ion battery \\
TOF & Time of fly \\
ESC & External short-circuits \\
SOH & State of health \\
EMA & Experimental modal analysis \\
US & Ultrasonic testing \\
AE & Acoustic emission \\
CEI & Cathode electrolyte interface \\
SEI & Solid electrolyte interface \\
EVs & Electric vehicles \\
NDT & Non-destructive testing \\
FFT & Fast Fourier transform \\
CAN & Controller area network \\
NCM & Nickel Manganese Cobalt Oxide
\end{tabular}

\section{References}

1. Wei, Z.; Zhao, J.; He, H.; Ding, G.; Cui, H.; Liu, L. Future smart battery and management: Advanced sensing from external to embedded multi-dimensional measurement. J. Power Sources 2021, 489, 229462. [CrossRef]

2. Wei, Z.; Zhao, J.; Ji, D.; Tseng, K.J. A multi-timescale estimator for battery state of charge and capacity dual estimation based on an online identified model. Appl. Energy 2017, 204, 1264-1274. [CrossRef]

3. Zhu, G.; Zhao, C.; Huang, J.; He, C.; Zhang, J.; Chen, S.; Xu, L.; Yuan, H.; Zhang, Q. Fast Charging Lithium Batteries: Recent Progress and Future Prospects. Small 2019, 15, 1805389. [CrossRef] [PubMed]

4. Tomaszewska, A.; Chu, Z.; Feng, X.; O'Kane, S.; Liu, X.; Chen, J.; Ji, C.; Endler, E.; Li, R.; Liu, L.; et al. Lithium-ion battery fast charging: A review. ETransportation 2019, 1, 100011. [CrossRef]

5. Liu, Y.; Zhu, Y.; Cui, Y. Challenges and opportunities towards fast-charging battery materials. Nat. Energy 2019, 4, 540-550. [CrossRef]

6. Birkl, C.R.; Roberts, M.R.; McTurk, E.; Bruce, P.G.; Howey, D.A. Degradation diagnostics for lithium ion cells. J. Power Sources 2017, 341, 373-386. [CrossRef] 
7. Chen, Z.; Zhou, N.; Liang, H.; Cui, J.; Fang, Z. A fusion-based method of state-of-charge online estimation for lithium-ion batteries under low capacity conditions. Front. Energy Res. 2021, 9, 797.

8. Divakaran, A.M.; Hamilton, D.; Manjunatha, K.N.; Minakshi, M. Design, development and thermal analysis of reusable Li-ion battery module for future mobile and stationary applications. Energies 2020, 13, 1477. [CrossRef]

9. $\quad$ Liu, D.; Shadike, Z.; Lin, R.; Qian, K.; Li, H.; Li, K.; Wang, S.; Yu, Q.; Liu, M.; Ganapathy, S.; et al. Review of recent development of in situ/operando characterization techniques for lithium battery research. Adv. Mater. 2019, 31, 1806620. [CrossRef]

10. Lu, J.; Wu, T.; Amine, K. State-of-the-art characterization techniques for advanced lithium-ion batteries. Nat. Energy 2017, 2, 17011. [CrossRef]

11. Wang, L.; Yin, S.; Zhang, C.; Huan, Y.; Xu, J. Mechanical characterization and modeling for anodes and cathodes in lithium-ion batteries. J. Power Sources 2018, 392, 265-273. [CrossRef]

12. Finegan, D.P.; Scheel, M.; Robinson, J.B.; Tjaden, B.; Di Michiel, M.; Hinds, G.; Brett, D.J.; Shearing, P.R. Investigating lithium-ion battery materials during overcharge-induced thermal runaway: An operando and multi-scale X-ray CT study. Phys. Chem. Chem. Phys. 2016, 18, 30912-30919. [CrossRef]

13. Heenan, T.M.; Tan, C.; Hack, J.; Brett, D.J.; Shearing, P.R. Developments in X-ray tomography characterization for electrochemical devices. Mater. Today 2019, 31, 69-85. [CrossRef]

14. Pfrang, A.; Kersys, A.; Kriston, A.; Sauer, D.U.; Rahe, C.; Käbitz, S.; Figgemeier, E. Geometrical in homogeneities as cause of mechanical failure in commercial 18,650 lithium ion cells. J. Electrochem. Soc. 2019, 166, A3745. [CrossRef]

15. Wu, Y.; Saxena, S.; Xing, Y.; Wang, Y.; Li, C.; Yung, W.K.; Pecht, M. Analysis of manufacturing-induced defects and structural deformations in lithium-ion batteries using computed tomography. Energies 2018, 11, 925. [CrossRef]

16. Robinson, J.B.; Engebretsen, E.; Finegan, D.P.; Darr, J.; Hinds, G.; Shearing, P.R.; Brett, D.J. Detection of internal defects in lithium-ion batteries using lock-in thermography. ECS Electrochem. Lett. 2015, 4, A106. [CrossRef]

17. Friesen, A.; Schultz, C.; Brunklaus, G.; Rodehorst, U.; Wilken, A.; Haetge, J.; Winter, M.; Schappacher, F. Long term aging of automotive type lithium-ion cells. ECS Trans. 2015, 69, 89. [CrossRef]

18. Tsutsui, W. The Mechanical Response of Multifunctional Battery Systems. Ph.D. Thesis, Purdue University, West Lafayette, IN, USA, 2017.

19. Gao, X.; He, P.; Ren, J.; Xu, J. Modeling of contact stress among compound particles in high energy lithium-ion battery. Energy Storage Matter. 2019, 18, 23-33. [CrossRef]

20. Zhu, J.E.; Li, W.; Xia, Y.; Sahraei, E. Testing and modeling the mechanical properties of the granular materials of graphite anode. J. Electrochem. Soc. 2018, 165, A1160-A1168. [CrossRef]

21. Higa, K.; Srinivasan, V. Stress and strain in silicon electrode models. J. Electrochem. Soc. 2015, 162, A1111-A1122. [CrossRef]

22. Cannarella, J.; Arnold, C.B. The effects of defects on localized plating in lithium-ion batteries. J. Electrochem. Soc. 2015, 162, A1365. [CrossRef]

23. Etiemble, A.; Idrissi, H.; Meille, S.; Roué, L. In situ investigation of the volume change and pulverization of hydride materials for Ni-MH batteries by concomitant generated force and acoustic emission measurements. J. Power Sources 2012, 205, 500-505. [CrossRef]

24. Majasan, J.; Robinson, J.; Owen, R.; Maier, M.; Radhakrishnan, A.N.P.; Pham, M.; Tranter, T.G.; Zhang, Y.; Shearing, P.; Brett, D. Recent advances in acoustic diagnostics for electrochemical power systems. J. Phys. Energy 2021, 3, 032011. [CrossRef]

25. Woodford, W.H.; Chiang, Y.; Carter, W.C. Quantifying reliability statistics for electrochemical shock of brittle materials. J. Mech. Phys. Solids 2014, 70, 71-83. [CrossRef]

26. Nakamura, H.; Ohtsu, M.; Enoki, M.; Mizutani, Y.; Shigeishi, M.; Inaba, H.; Nakano, M.; Shiotani, T.; Yuyama, S.; Sugimoto, S Practical Acoustic Emission Testing; Springer: Tokyo, Japan, 2016.

27. Kircheva, N.; Genies, S.; Brun-Buisson, D.; Thivel, P.X. Study of solid electrolyte interface formation and lithium intercalation in Li-Ion batteries by acoustic emission. J. Electrochem. Soc. 2011, 159, A18. [CrossRef]

28. Popp, H.; Koller, M.; Jahn, M.; Bergmann, A. Mechanical methods for state determination of Lithium-Ion secondary batteries: A review. J. Energy Storage 2020, 32, 101859. [CrossRef]

29. Kim, C.S.; Yoo, J.S.; Jeong, K.M.; Kim, K.; Yi, C.W. Investigation on internal short circuits of lithium polymer batteries with a ceramic-coated separator during nail penetration. J. Power Sources 2015, 289, 41-49. [CrossRef]

30. Choe, C.Y.; Jung, W.S.; Byeon, J.W. Damage evaluation in lithium cobalt oxide/carbon electrodes of secondary battery by acoustic emission monitoring. Mater. Trans. 2015, 56, 269-273. [CrossRef]

31. Villevieille, C.; Boinet, M.; Monconduit, L. Direct evidence of morphological changes in conversion type electrodes in Li-ion battery by acoustic emission. Electrochem. Commun. 2010, 12, 1336-1339. [CrossRef]

32. Divakaran, A.M.; Minakshi, M.; Bahri, P.A.; Paul, S.; Kumari, P.; Divakaran, A.M.; Manjunatha, K.N. Rational design on materials for developing next generation lithium-ion secondary battery. Prog. Solid State Chem. 2021, 62, 100298. [CrossRef]

33. Bauermann, L.P.; Mesquita, L.V.; Bischoff, C.; Drews, M.; Fitz, O.; Heuer, A.; Biro, D. Scanning acoustic microscopy as a non-destructive imaging tool to localize defects inside battery cells. J. Power Sources Adv. 2020, 6, 100035. [CrossRef] 